\title{
Pien Tze Huang alleviates 5-fluorouracil-induced intestinal mucositis in CT-26 tumor-bearing mice
}

\author{
CAIXUAN FU ${ }^{1,2}$, JIANFENG CHU $^{1}$, ALING SHEN ${ }^{1,2}$, LIYA LIU $^{1,3}$, HONGWEI CHEN $^{1}$, \\ JIUMAO LIN ${ }^{1,2}$, THOMAS J. SFERRA ${ }^{1,3}$, YOUQIN CHEN ${ }^{1,3}$ and JUN PENG ${ }^{1,2}$ \\ ${ }^{1}$ Academy of Integrative Medicine; ${ }^{2}$ Fujian Key Laboratory of Integrative Medicine on Geriatrics, Fujian University
of Traditional Chinese Medicine, Fuzhou, Fujian 350122, P.R. China; ${ }^{3}$ Pediatric Department, Rainbow Babies
and Children's Hospital, Case Western Reserve University School of Medicine, Cleveland, OH 44106, USA
}

Received June 17, 2016; Accepted March 31, 2017

DOI: $10.3892 /$ etm.2017.4755

\begin{abstract}
Chemotherapeutic agents, including 5-fluorouracil (5-FU), frequently cause intestinal mucositis as a side effect, leading to life quality reduction in colorectal cancer (CRC) patients and interruption of CRC treatment. Traditional Chinese medicines (TCMs) have recently received attention due to their relatively few adverse effects. Pien Tze Huang (PZH), a well-known TCM formulation first documented to have been prescribed $>450$ years ago, has been demonstrated to be clinically effective in treating various types of cancer including CRC. Although previous studies by our group reported that $\mathrm{PZH}$ possesses a variety of anti-cancer activities via multiple mechanisms, it has remained elusive whether it is able to reduce intestinal mucositis induced by $5-\mathrm{FU}$. The present study evaluated the effect of $\mathrm{PZH}$ on 5-FU-induced intestinal mucositis in CT-26 tumor-bearing xenograft mice and investigated the possible molecular mechanism. The results indicated that administration of PZH effectively alleviated the severity of 5-FU-induced diarrhea and morphological intestinal damages, but had no significant effect on body weight loss. In addition, a terminal deoxynucleotidyl transferase dUTP nick end labeling assay revealed that PZH treatment significantly inhibited cell apoptosis in the intestinal crypt. Furthermore,
\end{abstract}

Correspondence to: Dr Youqin Chen, Pediatric Department, Rainbow Babies and Children's Hospital, Case Western Reserve University School of Medicine, 2109 Adelbert Road, Cleveland, OH 44106, USA

E-mail:yxc571@case.edu

Dr Jun Peng, Academy of Integrative Medicine, Fujian University of Traditional Chinese Medicine, 1 Qiuyang Road, Minhou Shangjie, Fuzhou, Fujian 350122, P.R. China

E-mail: pjunlab@hotmail.com

Abbreviations: PZH, Pien Tze Huang; CRC, colorectal cancer; 5-FU, 5-fluorouracil; TCM, Traditional Chinese Medicine

Key words: Pien Tze Huang, colorectal cancer, 5-fluorouracil, intestinal mucositis, apoptosis immunohistochemical staining showed that PZH treatment reduced the protein expression of pro-apoptotic B-cell lymphoma 2 (Bcl-2)-associated $\mathrm{X}$ protein but enhanced that of anti-apoptotic Bcl-2 in the intestinal crypt. Taken together, the results of the present study suggested that PZH effectively attenuates 5-FU-induced intestinal mucositis, which is in part associated with its inhibitory effect on cell apoptosis in the intestinal crypt.

\section{Introduction}

Colorectal cancer (CRC) poses a significant threat to human health and is the second most commonly diagnosed cancer in females and the third in males, with an estimated 1.4 million new cases and 693,900 deaths occurring in 2012 worldwide (1). The incidence of CRC is increasing due to smoking, lack of physical activity, overweight and obesity, unhealthy diet and excessive alcohol consumption (2). Chemotherapy is currently a major post-operative adjunctive or preferred therapy for patients with CRC in intermediate and advanced stages. 5-Fluorouracil (5-FU)-based chemotherapy is the most widely prescribed treatment for CRC in clinical practice (3). However, normal cells, tissues and their functions are inevitably injured by $5-\mathrm{FU}$, which disrupts the balance of the body's self-regulatory system $(4,5)$. The major side effects of 5-FU include intestinal mucositis, myelosuppression and immunosuppression (6). It has been reported that $\sim 80 \%$ of cancer patients who have received 5-FU treatment displayed clinical manifestations of intestinal mucositis, such as severe diarrhea, mal-absorption, vomiting and bleeding $(7,8)$. The side effect not only forces certain patients to completely abandon the treatments, but also markedly affects their quality of life.

The pathogenic mechanisms of 5-FU-induced intestinal mucositis are complex and heterogeneous, including direct toxicity, apoptosis, hypo-proliferation, oxidative stress and abnormal inflammation (9-11). In particular, cell apoptosis has a critical role in the intestinal mucositis induced by 5-FU. Clinical studies and animal experiments have demonstrated that a large amount of apoptotic cells were observed in the intestinal crypt prior to serious mucosal destruction after 5-FU treatment $(12,13)$. Apoptosis is triggered by proteolytic 
enzymes such as caspases, a family of cysteine proteases that act as common death effector molecules (14). Activation of caspases may be initiated through two signaling pathways, the intrinsic (mitochondrial) pathway and the extrinsic (death-receptor) pathway $(15,16)$. The intrinsic pathway is generally activated when pro-apoptotic proteins such as B-cell lymphoma 2 (Bcl-2)-associated $\mathrm{X}$ protein (Bax) are released from the mitochondria. In addition, it has been shown that apoptosis induced by $5-\mathrm{FU}$ at high doses $(130-200 \mathrm{mg} / \mathrm{kg})$ is accompanied by an alteration of Bax and Bcl-2 expression, indicating the activation of the intrinsic apoptotic pathway $(12,13)$.

Traditional Chinese Medicine (TCM), as an important complementary and alternative medicine, evolved over thousands of years with its own unique system of theories, diagnostics and therapies in China and Southeast Asia. TCM has a number of benefits, such as having relatively few side effects, the capacity to decrease negative side effects associated with chemotherapy, enhancement of the effects of conventional cancer treatments, and improvement of patients' quality of life and immune function (17). Therefore, TCM has been increasingly used in conjunction with chemotherapies to improve the clinical management of patients with cancer. Pien Tze Huang (PZH) is a well-known TCM formulation, the prescription of which was first documented by a royal physician $>450$ years ago during the Chinese Ming Dynasty. PZH has been used in China for centuries as a folk remedy for various cancer types, including CRC. Although previous studies by our group reported that $\mathrm{PZH}$ possesses a variety of anti-cancer activities via multiple mechanisms (18-30), it has remained elusive whether $\mathrm{PZH}$ reduces intestinal mucositis induced by 5-FU. The present study evaluated the effect of PZH on 5-FU-induced intestinal mucositis in CT-26 tumor-bearing xenograft mice and investigated the possible molecular mechanism.

\section{Materials and methods}

Materials and reagents. RPMI-1640 medium, fetal bovine serum (FBS), penicillin-streptomycin and trypsin-EDTA were purchased from Thermo Fisher Scientific, Inc. (Waltham, MA, USA). Matrigel was obtained from BD Biosciences (San Jose, CA, USA). Bcl-2 (cat. no. ART1069) and Bax (cat. no. ARE6007) antibodies were provided by Antibody Revolution Inc. (San Diego, CA, USA) and horseradish peroxidase (HRP)-conjugated secondary antibodies (cat. no. Kit-0017) were obtained from Maix in Corp. (Fuzhou, Fujian, China). Harris' hematoxylin was obtained from Solarbio Corp. (Beijing, China). A terminal deoxynucleotidyl transferase-mediated dUTP nick end labeling (TUNEL) assay kit was purchased from Keygen Biotech (Nanjing, China).

Preparation of $\mathrm{PZH}$. PZH was obtained from and authenticated by the sole manufacturer Zhangzhou Pien Tze Huang Pharmaceutical Co., Ltd. (Zhangzhou, China; Chinese Food and Drug Administration approval no. Z35020242). Stock solutions of $\mathrm{PZH}$ were prepared immediately prior to use by dissolving the $\mathrm{PZH}$ powder in saline to a concentration of $25 \mathrm{mg} / \mathrm{ml}$ and sonication for $30 \mathrm{~min}$. The sample was ready for intragastric infusion when the powder was completely dissolved and the solution became clear.

Preparation of 5-FU. 5-FU was obtained from the Xudong Haipu Pharmaceutical Co., Ltd. (Shanghai, China). Immediately prior to use, 5-FU was dissolved in saline to a concentration of $15 \mathrm{mg} / \mathrm{ml}$.

Cell culture. The CT-26 murine colon carcinoma cell line was purchased from the Shanghai Cell Bank of the Chinese Academy of Sciences. The cells were grown in RPMI-1640 medium containing $10 \%$ (v/v) FBS, $100 \mathrm{U} / \mathrm{ml}$ penicillin and $100 \mu \mathrm{g} / \mathrm{ml}$ of streptomycin in a humidified incubator at $37^{\circ} \mathrm{C}$ in an atmosphere containing $5 \% \mathrm{CO}_{2}$.

Animals. A total of 32 male BALB/c mice (initial body weight, 20-22 g, 4-6 weeks old), obtained from Shanghai SLAC Laboratory Animal Co., Ltd. (Shanghai, China), were allowed to acclimatize for two days and were housed in a specific pathogen-free environment with food and water supplied ad libitum throughout the experiment. The environment was maintained at $22^{\circ} \mathrm{C}$ with a 12 -h light/dark cycle. All the animal treatments were performed in compliance with international ethical guidelines and the National Institutes of Health Guidelines for the Care and Use of Laboratory Animals. The experiments were approved by the Institutional Animal Care and Use Committee of Fujian University of Traditional Chinese Medicine (Fuzhou, China).

Establishment of mice xenografted with CT-26. After detachment through trypsinization with trypsin-EDTA, CT-26 cells were re-suspended in serum-free RPMI-1640. A total of $2 \times 10^{6}$ cells mixed with Matrigel (1:1) were subcutaneously injected into the right flank region of each mouse. When the tumor size reached $300-350 \mathrm{~mm}^{3}$, the mice were randomly divided into 4 groups ( $\mathrm{n}=8$ in each): Control group, $\mathrm{PZH}$ group, 5-FU group and 5-FU + PZH group. $250 \mathrm{mg} / \mathrm{kg}$ of PZH was orally administered daily for 4 days, while 5-FU $(150 \mathrm{mg} / \mathrm{kg}$ ) was intraperitoneally administered only on the first day of treatment. The body weight was measured every day. At the end of the experiment, mice were sacrificed by cervical dislocation. The jejunum was excised for further analyses.

Mucositis assessment. Disease severity was assessed daily by monitoring body weight and scoring for diarrhea. Diarrhea score was calculated based on the consistency of stools, using the modified parameters as previously described (31): 0 , normal (normal stool or absent); 1 , slight (slightly wet and soft stool); 2, moderate (wet and unformed stool with moderate perianal staining of the coat); 3 , severe (watery stool with severe perianal staining of the coat).

Intestinal morphometry and histopathological assessment. A segment of the jejunum (3-cm segment immediately distal to the ligament of Treitz) was taken. Specimens were fixed in $4 \%$ paraformaldehyde for $24 \mathrm{~h}$, embedded in paraffin, sectioned at $4 \mu \mathrm{m}$ and stained with haematoxylin and eosin (H\&E). Morphological assessment was performed by measuring the change in jejunum villus height (from the top 


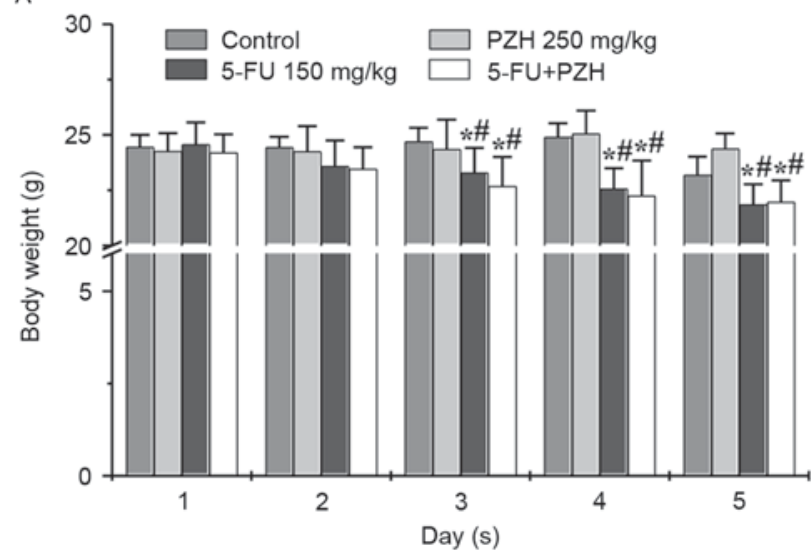

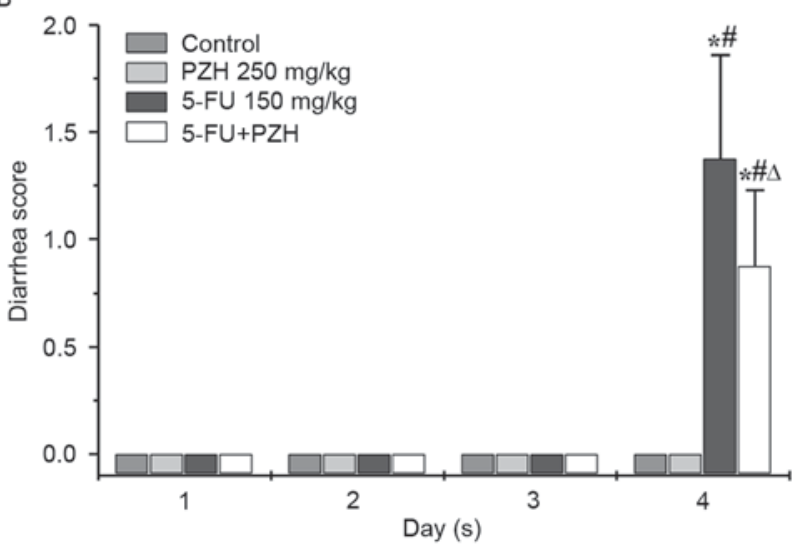

Figure 1. Effect of PZH on diarrhea induced by 5-FU in CT-26 tumor-bearing mice. Animals were administered 5-FU (150 mg/kg, intraperitoneally) once on day 1 and PZH (250 mg/kg per os) once daily from days 1-4. (A) Body weight was monitored daily and is shown as a percentage of initial body weight, whereas (B) the severity of diarrhea was scored daily using the four-grade scale $(0-3)$. Values are expressed as the mean \pm standard deviation $(\mathrm{n}=8$ per group). $\mathrm{P}<0.05$ vs. controls; ${ }^{\#} \mathrm{P}<0.05$ vs. PZH; ${ }^{\Delta} \mathrm{P}<0.05$ vs. 5-FU. PZH, Pien Tze Huang; 5-FU, 5 -fluorouracil.

of the villus to the villus-crypt junction) and crypt depth (defined as invagination depth between adjacent villi) using a light microscope and images were captured at a magnification of $x 100$ and $x 400$. The shortening of villi was evaluated by the average villus-to-crypt ratio. Ten intact and well-oriented villi and crypts were measured and averaged in longitudinal tissue sections for each sample.

Histopathological assessment was performed by using a modification of the histopathological scores described by Macpherson and Pfeiffer (32) and was graded as follows: 0 , normal histological findings; 1, mucosa: Villus blunting, loss of crypt architecture, sparse inflammatory cell infiltration, vacuolization and edema, but normal muscular layer; 2, mucosa: Villus blunting with fattened and vacuolated cells, crypt necrosis, intense inflammatory cell infiltration, vacuolization and edema, but normal muscular layer; 3, mucosa: Villus blunting with fattened and vacuolated cells, crypt necrosis, intense inflammatory cell infiltration, vacuolization and edema, and muscular layer: Edema, vacuolization, sparse neutrophil infiltration.

TUNEL assay. The 4- $\mu \mathrm{m}$-thick sections of jejunum samples were analyzed by TUNEL staining using an in situ apoptosis detection kit. Apoptotic cells were counted as diaminobenzidine (DAB)-positive cells (brown staining) at five arbitrarily selected microscopic fields at a magnification of x400 under a light microscope.

Immunohistochemistry (IHC) assay. IHC staining for Bcl-2 and Bax was performed to assess apoptotic signaling. In brief, the jejunum samples were fixed with $4 \%$ paraformaldehyde for $24 \mathrm{~h}$, embedded in paraffin and cut into $4-\mu \mathrm{m}$ sections. The sections were subjected to antigen retrieval and the endogenous peroxidase activity was quenched with hydrogen peroxide. After blocking non-specific proteins, the sections were incubated at $4^{\circ} \mathrm{C}$ overnight with primary antibodies against $\mathrm{Bcl}-2$ (dilution, 1:400) and Bax (dilution, 1:400). After being washed with PBS, the slides were stained with a HRP-conjugated secondary antibodies and then washed with PBS. The slides were then incubated with DAB as the chromogen, followed by counterstaining with diluted Harris' hematoxylin. After staining, five high-power fields (magnification, x400) were randomly selected for each slide and the average proportion of Bcl-2 and Bax-positive cells in each field was counted using the True Color Multi-Functional Cell Image Analysis Management System (Image-Pro Plus; Media Cybernetics, Rockville, MD, USA). To exclude any non-specific staining, PBS was used to replace the primary antibody as a negative control.

Statistical analysis. Values are expressed as the mean \pm standard error of the mean of six animals per group and were analyzed using the SPSS package for Windows (version 18.0; SPSS, Inc., Chicago, IL, USA). Statistical analysis of the data was performed with Student's t-test and analysis of variance. $\mathrm{P}<0.05$ was considered to indicate a statistically significant difference.

\section{Results}

PZH alleviates 5-FU-induced diarrhea in CT-26 tumor-bearing mice. Since body weight is a widely used indicator and diarrhea is the most common clinical symptom for the evaluation of 5-FU-induced intestinal mucositis $(33,34)$, the present study assessed body weight changes and diarrhea in CT-26 xenograft mice after treatment with 5-FU and/or PZH. As shown in Fig. 1A, 5-FU challenge significantly reduced the body weight in tumor-bearing mice as compared with that in the controls and caused serious diarrhea. Although PZH did not neutralize body weight loss caused by 5-FU, it significantly alleviated 5-FU-induced diarrhea in the experimental animals (Fig. 1B). PZH alone did not result in body weight loss and diarrhea during the course of the experiment.

PZH ameliorates 5-FU-induced histopathological changes in the intestine of CT-26 tumor-bearing mice. The histopathological changes in jejunum tissues were examined by $\mathrm{H} \& \mathrm{E}$ staining. As presented in Fig. 2A, compared with mice in the control or PZH only group, 5-FU-treated mice displayed severe tissue damage in the jejunum, including villus deformation, 
Control

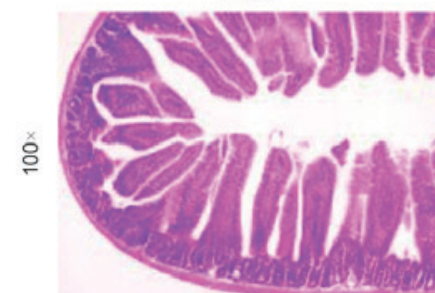

$\mathrm{PZH}$
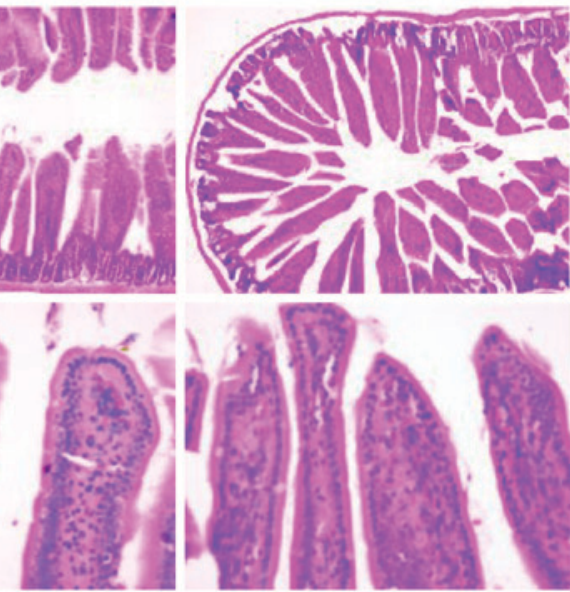
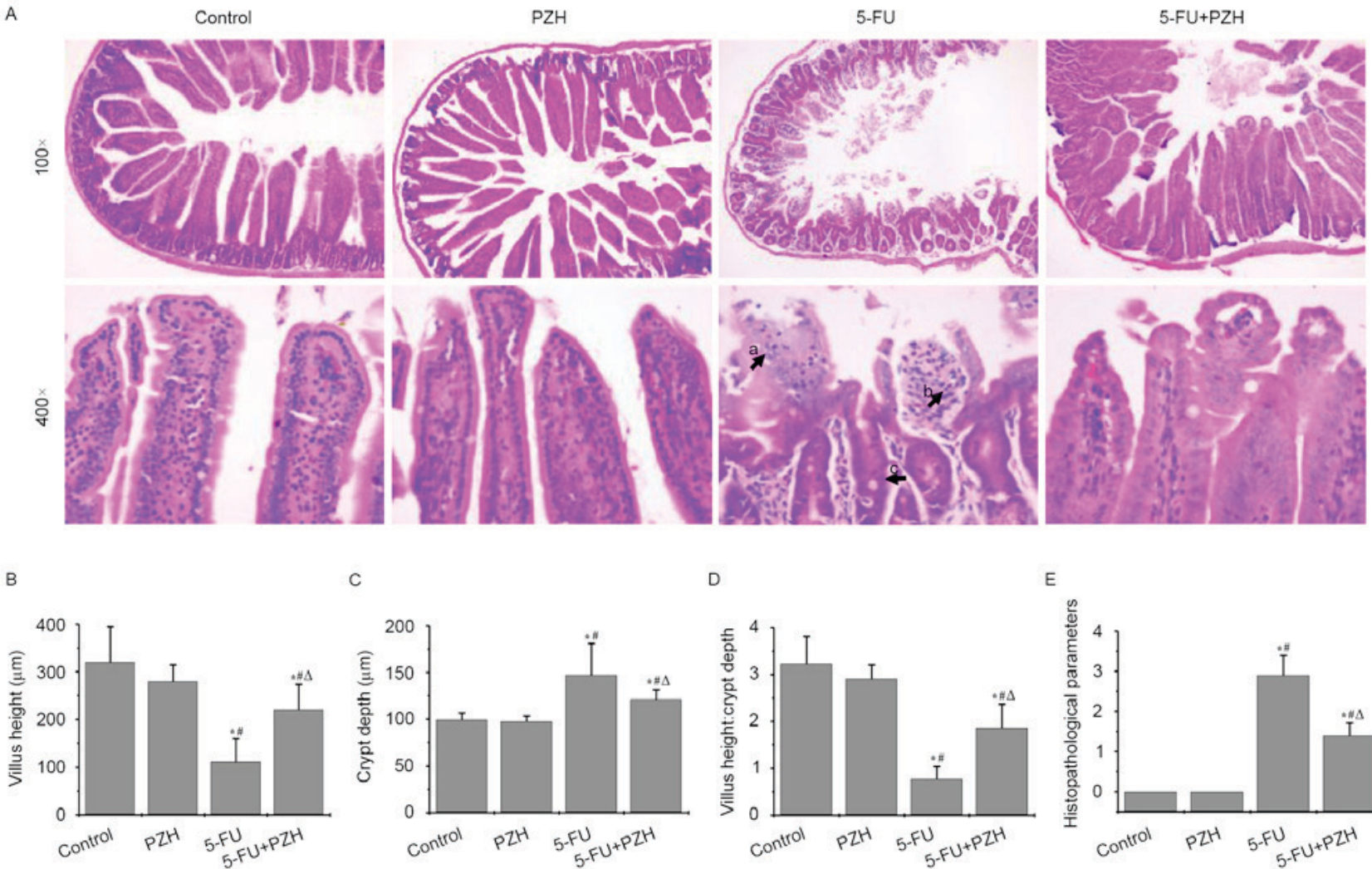

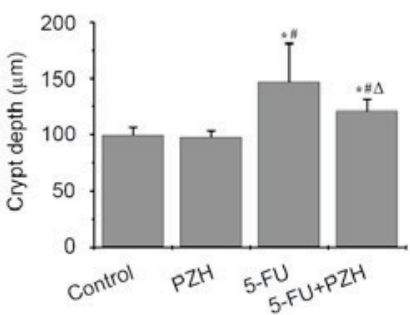

D

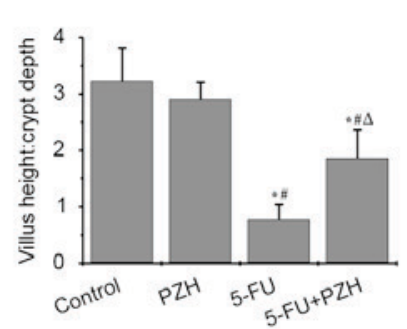

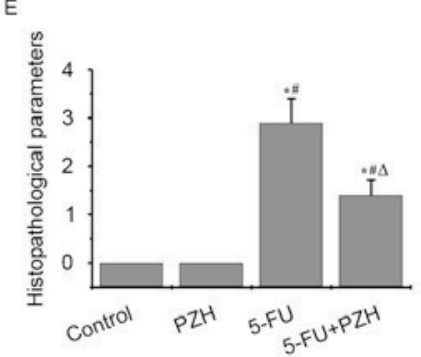

Figure 2. Effect of PZH on intestinal mucositis induced by 5-FU in CT-26 tumor-bearing mice. (A) Hematoxylin and eosin staining was used to visualize the formalin-fixed sections of the jejunum (magnification, x100 and x400 as indicated). Mice with 5-FU-induced intestinal mucositis displayed (a) shortened villi with fattened and vacuolated cells, (b) inflammatory cell infiltration in the lamina propria and (c) loss of normal crypt architecture (indicated by arrowheads). (B-D) Morphological assessment was performed by measuring changes in (B) the jejunum villus height, (C) crypt depth and (D) the ratio of villus height and crypt depth under a light microscope. (E) Histopathological parameters were assessed by using a modification of the histopathological grading system by Macpherson and Pfeiffer. Values are expressed as the mean \pm standard deviation $\left(\mathrm{n}=8\right.$ per group). ${ }^{*} \mathrm{P}<0.05$ vs. controls; ${ }^{\#} \mathrm{P}<0.05$ vs. $\mathrm{PZH} ;{ }^{\Delta} \mathrm{P}<0.05$ vs. 5 -FU. PZH, Pien Tze Huang; 5-FU, 5-fluorouracil.

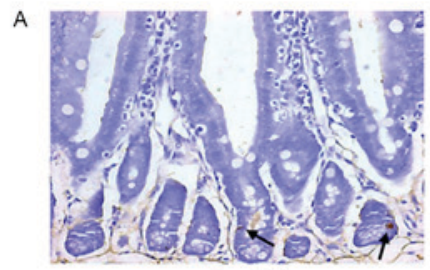

Control

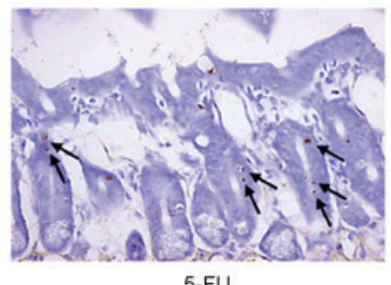

5-FU

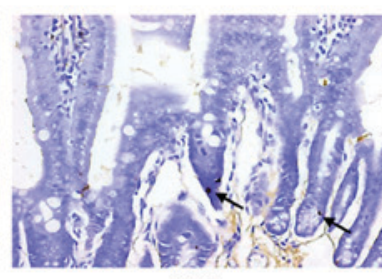

$\mathrm{PZH}$

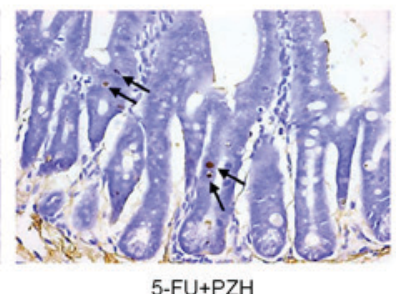

B

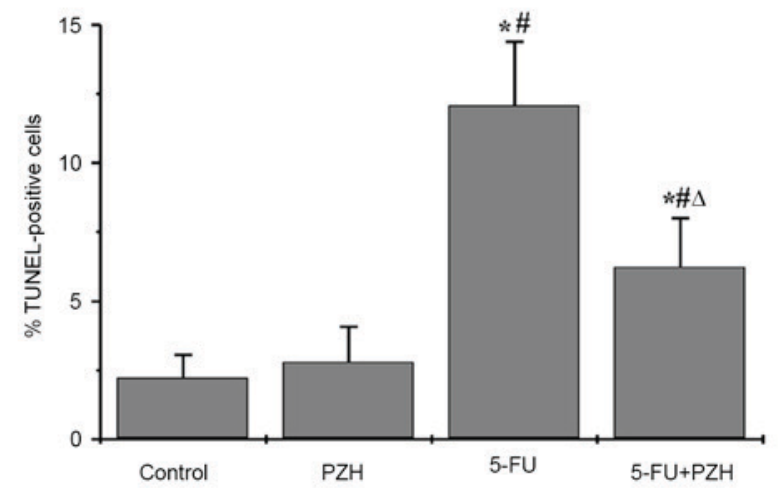

Figure 3. Effect of PZH on apoptosis induced by 5-FU in intestinal crypt cells. At the end of the experiment, the jejunum tissues from the four groups were processed for TUNEL assay. (A) Representative images are shown (magnification, x400). The black arrowheads indicate TUNEL-positive cells. (B) Quantification of apoptosis was performed by determining the percentage of TUNEL-positive cells. Values are expressed as the mean \pm standard deviation ( $\mathrm{n}=8$ per group). ${ }^{*} \mathrm{P}<0.05$ vs. controls; ${ }^{\#} \mathrm{P}<0.05$ vs. $\mathrm{PZH} ;{ }^{\Delta} \mathrm{P}<0.05$ vs. 5-FU. PZH, Pien Tze Huang; 5-FU, 5-fluorouracil; TUNEL, terminal deoxynucleotidyl transferase dUTP nick end labeling.

loss and atrophy, loss of normal crypt architecture, intense inflammatory cell infiltration, vacuolization and oedema (arrows). However, administration of PZH significantly ameliorated 5-FU-induced intestinal damage in tumor-bearing mice (Fig. 2B-D). These observations were verified by histopathological assessment, where the microscopic scores after 


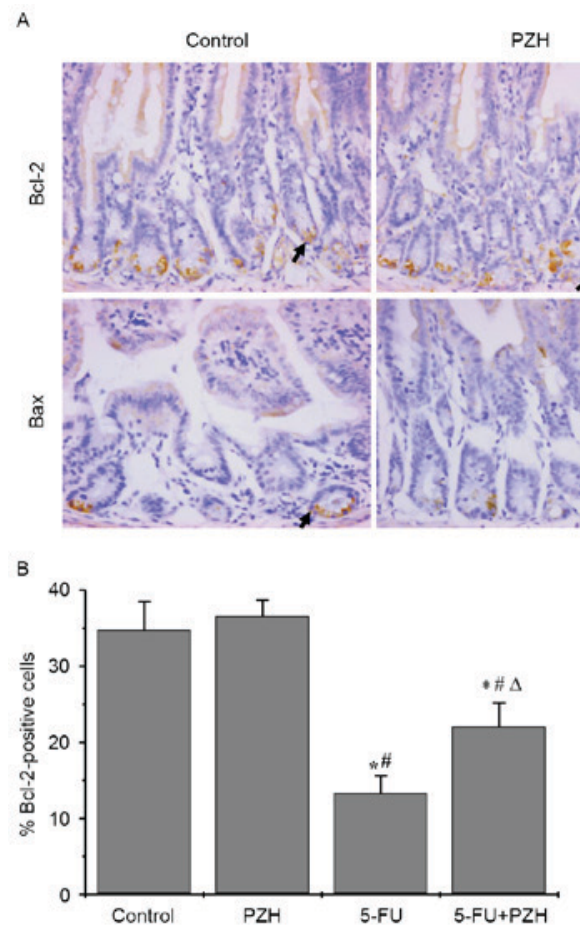

$\mathrm{PZH}$

5-FU

$5-\mathrm{FU}+\mathrm{PZH}$
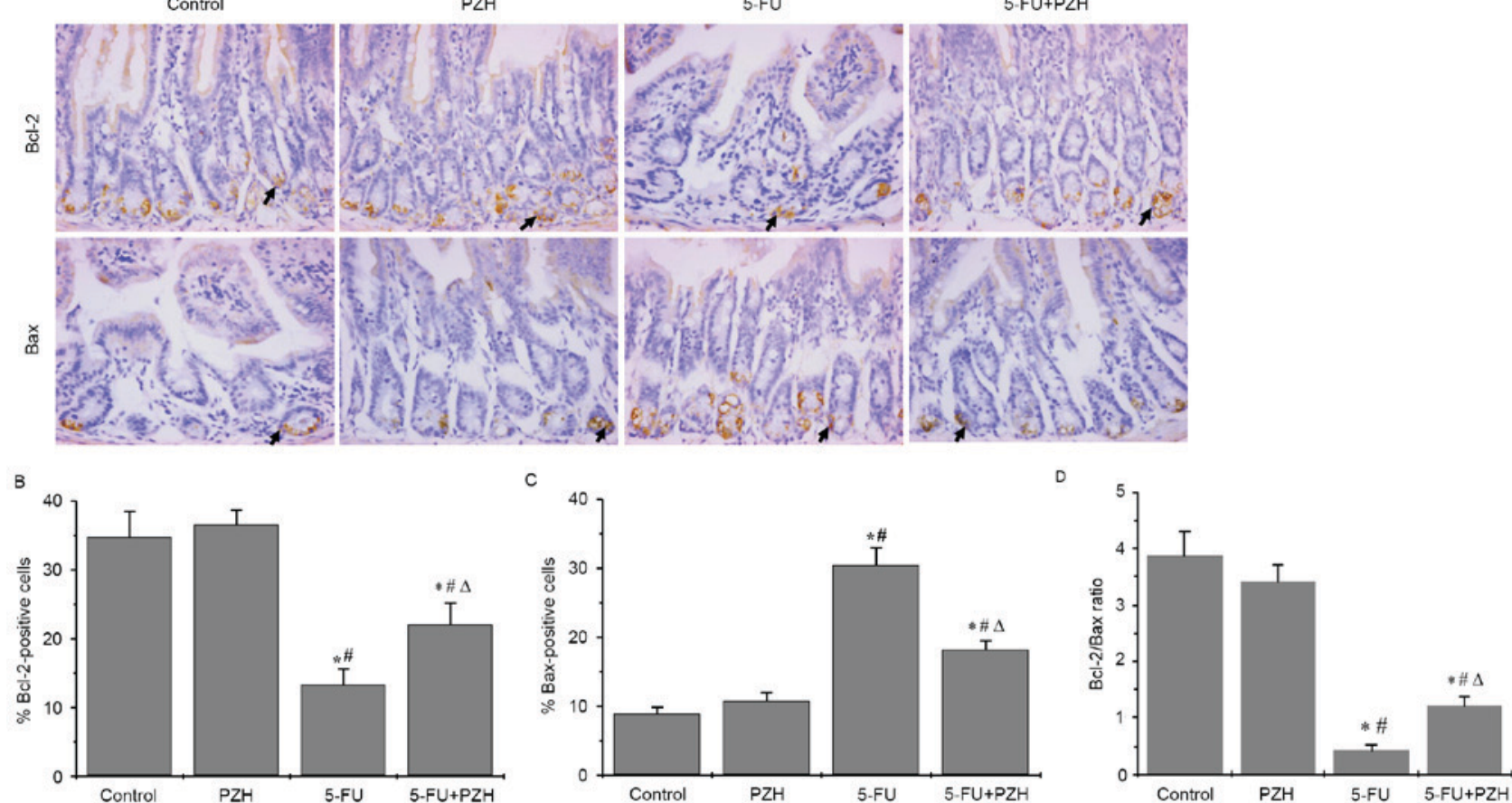

Figure 4. Effect of PZH on the expression of Bcl-2 and Bax induced by 5-FU in intestinal crypt cells. At the end of the experiment, the jejunum tissues from the four groups were processed for immunohistochemical staining for Bcl-2 or Bax. (A) Representative images are shown (magnification, $\mathrm{x} 400$ ). The black arrowheads indicate positive staining for Bcl-2 and Bax. (B and C) Quantification of the percentage of cells stained for Bcl-2 and Bax, respectively. (D) Bcl-2/Bax ratio. Values are expressed as the mean \pm standard deviation ( $\mathrm{n}=8$ per group). ${ }^{*} \mathrm{P}<0.05$ vs. controls; ${ }^{\#} \mathrm{P}<0.05$ vs. $\mathrm{PZH} ;{ }^{\Delta} \mathrm{P}<0.05$ vs. 5 -FU. $\mathrm{PZH}, \mathrm{Pien} \mathrm{Tze} \mathrm{Huang}$; 5-FU, 5-fluorouracil; Bcl-2, B-cell lymphoma 2; Bax, Bcl-2-associated X protein.

administration of 5-FU with or without $\mathrm{PZH}$ were 2.9 and 1.4 , respectively $(\mathrm{P}<0.05$; Fig. $2 \mathrm{E})$.

PZH inhibits 5-FU-induced apoptosis of intestinal crypt cells in CT-26 tumor-bearing mice via modulation of Bcl-2 and Bax protein expression. Apoptosis is the critical factor in the occurrence of intestinal mucositis induced by 5-FU (22); therefore, a TUNEL assay was performed in order to analyze the effect of PZH on the apoptosis of intestinal crypt cells induced by 5-FU. As presented in Fig. 3, 5-FU challenge significantly increased the percentage of TUNEL-positive intestinal crypt cells in xenograft mice, as compared with that in the mice in the control or PZH only groups, which, however, was significantly reduced by $\mathrm{PZH}$ treatment. In addition, IHC staining revealed that 5-FU significantly reduced the level of the anti-apoptotic protein Bcl-2 in intestinal crypt cells, whereas the level of the pro-apoptotic protein Bax was significantly increased after 5-FU challenge (Fig. 4A-C). However, 5-FU-induced imbalance of the $\mathrm{Bcl}-2 / \mathrm{Bax}$ ratio was reversed by PZH treatment (Fig. 4D).

\section{Discussion}

5-FU is one of the anti-cancer drugs widely utilized in the treatment of CRC in clinical practice (6). However, continuous use of 5-FU usually has severe toxic effects, particularly intestinal mucositis (12), resulting in discontinuation of cancer chemotherapy and impairment of the quality of life of patients. Therefore, immediate and effective countermeasures to overcome these potential problems are urgently required. TCMs have attracted an increasing amount of attention worldwide, since they have a relatively low toxicity as compared with that of modern chemotherapeutics and have a long history of application in cancer therapy. PZH, a well-known TCM formulation, has long been used as a folk remedy for various cancer types in China and Southeast Asia. Previous studies by our group have demonstrated that PZH possesses a broad range of anti-cancer activities due to its ability to affect multiple intracellular targets, suggesting that $\mathrm{PZH}$ may be a novel multi-potent therapeutic agent for the treatment of CRC. In order to further elucidate the beneficial effects of $\mathrm{PZH}$ in cancer therapy, the present study evaluated its effect on intestinal mucositis induced by a single dose of 5-FU using a CT-26 CRC xenograft mouse model.

Consistent with the results of previous studies (10-12), administration of 5-FU to mice caused severe intestinal mucositis, which was morphologically characterized by the shortening of villi, destruction of intestinal crypts and intense inflammatory cell infiltration, accompanied by clinical symptoms such as diarrhea and body weight loss. More importantly, it was demonstrated that $\mathrm{PZH}$ treatment alleviated the severity of 5-FU-induced morphological intestinal damages and diarrhea, although it had no significant effect on body weight loss.

The pathogenesis of 5-FU-induced intestinal mucositis is remains to be fully elucidated; however, it is considered to be a consequence of several pathogenic elements, including direct toxicity, oxidative stress, apoptosis, hypo-proliferation and abnormal inflammation (19-21). In particular, apoptosis is the critical factor in the occurrence of intestinal mucositis induced 
by 5 -FU (22). Several studies have indicated that apoptosis of intestinal crypt cells induced by 5-FU is accompanied by increased pro-apoptotic Bax expression and decreased anti-apoptotic Bcl-2 expression $(22,23)$. As expected, the present study we observed that 5-FU challenge significantly promoted cell apoptosis in the intestinal crypt, with the increase of Bax expression and the decrease of Bcl-2 expression. However, administration of PZH inhibited 5-FU-induced apoptosis of intestinal crypt cells through suppression the pro-apoptotic $\mathrm{Bax} / \mathrm{Bcl}-2$ ratio.

The aim of this study was to investigate the effect and possible molecular mechanism of $\mathrm{PZH}$ on 5-FU-induced intestinal mucositis in CRC tumor-bearing xenograft mice. Meanwhile we observed the changes of tumor volume and weight. The results indicated that compared with the control group, PZH has no significant effect on tumor growth in CRC mice. A previous study by the current authors demonstrated that administration of PZH significantly inhibited CRC growth in vivo when the treatment period of $\mathrm{HZH}$ was more than 2 weeks, and the tumor inhibition occurred after 7 days of PZH administration (23). In the current study, PZH administration lasted only 5 days. Therefore, PZH didn't show inhibitory effect on the growth of CT-26 cell xenograft, but this have been associated with the shorter administration time of $\mathrm{PZH}$.

In conclusion, the findings of the present study suggested that that PZH may be a promising candidate for an adjuvant drug to help ameliorate the adverse effects of currently used modern chemotherapeutics.

\section{Acknowledgements}

This study was financially supported by the National Natural Science Foundation of China (no. 81403390), the Research Fund of the Education Bureau of Fujian Province (no. JA14162), the Joint Project of Education and Health Bureaus of Fujian Province (no. WKJ-FJ-21) and the Developmental Fund of Chen Keji Integrative Medicine (nos. CKJ2013012, CKJ2014004 and CKJ2015005).

\section{References}

1. Torre LA, Bray F, Siegel RL, Ferlay J, Lortet-Tieulent J and Jemal A Global cancer statistics, 2012. CA Cancer J Clin 65: 87-108, 2015.

2. Vineis P and Wild CP: Global cancer patterns: Causes and prevention. Lancet 383: 549-557, 2014.

3. Carrillo E, Navarro SA, Ramírez A, García MÁ, Griñán-Lisón C, Perán M and Marchal JA: 5-Fluorouracil derivatives: A patent review (2012-2014). Expert Opin Ther Pat 25: 1131-1144, 2015.

4. De Rosa M, Pace U, Rega D, Costabile V, Duraturo F, Izzo P and Delrio P: Genetics, diagnosis and management of colorectal cancer (Review). Oncol Rep 34: 1087-1096, 2015.

5. Longley DB, Allen WL and Johnston PG: Drug resistance, predictive markers and pharmacogenomics in colorectal cancer. Biochim Biophys Acta 1766: 184-196, 2006.

6. Chang CT, Ho TY, Lin H, Liang JA, Huang HC, Li CC, Lo HY, Wu SL, Huang YF and Hsiang CY: 5-Fluorouracil induced intestinal mucositis via nuclear factor- $\mathrm{\kappa B}$ activation by transcriptomic analysis and in vivo bioluminescence imaging. PLoS One 7: e31808, 2012.

7. Sonis ST, Elting LS, Keefe D, Peterson DE, Schubert M, Hauer-Jensen M, Bekele BN, Raber-Durlacher J, Donnelly JP, Rubenstein EB, et al: Perspectives on cancer therapy-induced mucosal injury: Pathogenesis, measurement, epidemiology, and consequences for patients. Cancer 100 (9 Suppl): S1995-S2025, 2004

8. Keefe DM: Gastrointestinal mucositis: A new biological model Support Care Cancer 12: 6-9, 2004.
9. Bowen JM, Gibson RJ, Cummins AG and Keefe DM: Intestinal mucositis: The role of the Bcl-2 family, p53 and caspases in chemotherapy-induced damage. Support Care Cancer 14: 713-731, 2006.

10. Daniele B, Secondulfo M, De Vivo R, Pignata S, De Magistris L, Delrio $\mathrm{P}$, Palaia R, Barletta E, Tambaro R and Carratù R: Effect of chemotherapy with 5-fluorouracil on intestinal permeability and absorption in patients with advanced colorectal cancer. J Clin Gastroenterol 32: 228-230, 2001.

11. Yasuda M, Kato S, Yamanaka N, Imori M, Utsumi D, Kitahara Y, Iwata K, Matsuno K, Amagase K, Yabe-Nishimura C and Takeuchi K: Potential role of the NADPH oxidase NOX1 in the pathogenesis of 5-fluorouracil-induced intestinal mucositis in mice. Am J Physiol Gastrointest Liver Physiol 302: G1133-G1142, 2012.

12. Wu ZQ, Han XD, Wang Y, Yuan KL, Jin ZM, Di JZ, Yan J, Pan Y, Zhang P, Huang XY, et al: Interleukin-1 receptor antagonist reduced apoptosis and attenuated intestinal mucositis in a 5 -fluorouracil chemotherapy model in mice. Cancer Chemother Pharmacol 68: 87-96, 2011.

13. Inomata A, Horii I and Suzuki K: 5-Fluorouracil-induced intestinal toxicity: What determines the severity of damage to murine intestinal crypt epithelia? Toxicol Lett 133: 231-240, 2002.

14. Peng J, Ding JZ, Tan CB, Baggenstoss B, Zhang Z, Lapolla SM and Lin J: Oligomerization of membrane-bound Bcl-2 is involved in its pore formation induced by tBid. Apoptosis 14: 1145-1153, 2009.

15. Fulda S and Debatin KM: Extrinsic versus intrinsic apoptosis pathways in anticancer chemotherapy. Oncogene 25: 4798-4811, 2006.

16. Peng J, Tan C, Roberts GJ, Nikolaeva O, Zhang Z, Lapolla SM, Primorac S, Andrews DW and Lin J: tBID elicits a conformational alteration in membrane-bound $\mathrm{Bcl}-2$ such that it inhibits Bax Pore formation. J Biol Chem 281: 35802-35811, 2006.

17. Li X, Yang G, Li X, Zhang Y, Yang J, Chang J, Sun X, Zhou X, Guo Y, Xu Y, et al: Traditional Chinese medicine in cancer care: A review of controlled clinical studies published in Chinese. PLoS One 8: e60338, 2013.

18. Lin JM, Wei LH, Chen YQ, Liu XX, Hong ZF, Sferra TJ and Peng J: Pien Tze Huang induced apoptosis in human colon cancer HT-29 cells is associated with regulation of the Bcl-2 family and activation of caspase-3. Chin J Integr Med 17: 685-690, 2011.

19. Zhuang Q, Hong F, Shen A, Zheng L, Zeng J, Lin W, Chen Y, Sferra TJ, Hong Z and Peng J: Pien Tze Huang inhibits tumor cell proliferation and promotes apoptosis via suppressing the STAT3 pathway in colorectal cancer mouse. Int J Oncol 40: 1569-1574, 2012.

20. Shen AL, Hong F, Liu LY, Lin JM, Zhuang QC, Hong ZF and Peng J: Effects of Pien Tze Huang on angiogenesis in vivo and in vitro. Chin J Integr Med 18: 431-436, 2012.

21. Shen A, Hong F, Liu L, Lin J, Wei L, Cai Q, Hong Z and Peng J: Pien Tze Huang inhibits the proliferation of human colon carcinoma cells by arresting G1/S cell cycle progression. Oncol Lett 4: 767-770, 2012.

22. Shen A, Chen Y, Hong F, Lin J, Wei L, Hong Z, Sferra TJ and Peng J: Pien Tze Huang suppresses IL-6-inducible STAT3 activation in human colon carcinoma cells through induction of SOCS3. Oncol Rep 28: 2125-2130, 2012.

23. Shen A, Lin J, Chen Y, Lin W, Liu L, Hong Z, Sferra TJ and Peng J: Pien Tze Huang inhibits tumor angiogenesis in a mouse model of colorectal cancer via suppression of multiple cellular pathways. Oncol Rep 30: 1701-1706, 2013.

24. Lin W, Zhuang Q, Zheng L, Cao Z, Shen A, Li Q, Fu C, Feng J and Peng J: Pien Tze Huang inhibits liver metastasis by targeting TGF- $\beta$ signaling in an orthotopic model of colorectal cancer. Oncol Rep 33: 1922-1928, 2015.

25. Wei L, Chen P, Chen Y, Shen A, Chen H, Lin W, Hong Z, Sferra TJ and Peng J: Pien Tze Huang suppresses the stem-like side population in colorectal cancer cells. Mol Med Rep 9: 261-266, 2014.

26. Chen H, Shen A, Zhang Y, Chen Y, Lin J, Lin W, Sferra T and Peng J: Pien Tze Huang inhibits hypoxia-induced epithelial-mesenchymal transition in human colon carcinoma cells through suppression of the HIF-1 pathway. Exp Ther Med 7: 1237-1242, 2014

27. Shen A, Lin W, Chen Y, Liu L, Chen H, Zhuang Q, Lin J, Sferra TJ and Peng J: Pien Tze Huang inhibits metastasis of human colorectal carcinoma cells via modulation of TGF- $\beta 1 / \mathrm{ZEB} / \mathrm{miR}$ 200 signaling network. Int J Oncol 46: 685-690, 2015. 
28. Chen H, Feng J, Zhang Y, Shen A, Chen Y, Lin J, Lin W, Sferra TJ and Peng J: Pien Tze Huang inhibits hypoxia-induced angiogenesis via HIF-1 $\alpha$ /VEGF-A pathway in colorectal cancer. Evid Based Complement Alternat Med 2015: 454279, 2015.

29. Shen A, Chen H, Chen Y, Lin J, Lin W, Liu L, Sferra TJ and Peng J: Pien Tze Hung overcomes multidrug resistance and epithelial-mesenchymal transition in human colorectal carcinoma cells via suppression of TGF- $\beta$ pathway. Evid Based Complement Alternat Med 2014: 679436, 2014.

30. Qi F, Wei L, Shen A, Chen Y, Lin J, Chu J, Cai Q, Pan J and Peng J: Pien Tze Huang inhibits the proliferation, and induces the apoptosis and differentiation of colorectal cancer stem cells via suppression of the Notch1 pathway. Oncol Rep 35: 511-517, 2016.
31. Yasuda M, Kato S, Yamanaka N, Iimori M, Matsumoto K, Utsumi D, Kitahara Y, Amagase K, Horie S and Takeuchi K: 5-HT3 receptor antagonists ameliorate 5-fluorouracil-induced intestinal mucositis by suppression of apoptosis in murine intestinal crypt cells. Br J Pharmacol 168: 1388-1400, 2013.

32. MacPherson BR and Pfeiffer CJ: Experimental production of diffuse colitis in rats. Digestion 17: 135-150, 1978.

33. Gibson RJ and Stringer AM: Chemotherapy-induced diarrhea. Curr Opin Support Palliat Care 3: 31-35, 2009.

34. Gibson RJ and Keefe DM: Cancer chemotherapy-induced diarrhoea and constipation: Mechanisms of damage and prevention strategies. Support Care Cancer 14: 890-900, 2006. 